\title{
Discussion to papers of Jesel et al., Jacobs and Meyer et al.
}

\author{
Chairman: DR J. Young
}

DR F. MEINECKe (Germany). Firstly, I would comment on the first paper presented by the Strasbourg group. I feel this paper confirms the statements made by Hardy and Rossier some years ago about the different possibilities of spinal cord and peripheral nerve lesions at the sacro-lumbar junction. Then on Meyer's paper, regarding closed reduction. The method you are using is different from that described by Laurenz Böhler. I used this method for many years, we don't press on the gibbus but we pull the patient up after he has been stretched in a long direction, so I feel one should mention that it is dangerous because you may press some bony fragment of the arch into the spinal canal and increase the spinal cord lesion.

DR P. MEYER (U.S.A.). I do not question the concern, because it is well described in the literature and I have reported it. We have on about three occasions had patients who had multiple fractures and multiple trauma requiring splenectomy, long bone debridement for open fractures and facial injuries who have been operated on by plastic and other surgeons all at the same time. In those patients we treated the patients by lifting while they were in the supine position and under general anaesthesia and we have been equally successful in that; but the use of that procedure has been indicated less than in patients who arrive in the emergency room and who have a significant amount of muscle spasm and require more traction, which is more easily performed in a prone position. It also makes it easier to obtain cross-table lateral X-rays when the patient's spine is away from the frame and you are confusing what is frame and what is posterior vertebral column. Not an excuse but an explanation.

PROFESSOR WeIsS (Poland). I would like to give my surgical remarks on both papers. It is quite impossible to use a straight Harrington rod without first correcting the kyphosis. You said you bent the Harrington rod according to the shape of the kyphosis but what happened later to the Harrington rods? We have tried to construct better hooks situated under report and have the possibility of overcoming this kyphosis. This shows that the springs which are placed in the space between the spinos and transverse processes are not doing any good. The one stage manipulation is much better and more often indicated than the Harrington rods. I saw your slides and I was surprised, because I have in my material been using the Lorenz Böhler technique, two cases which became much worse after a very quick laminectomy to remove the bone fragments.

DR JACOBS (U.S.A.). I think the technique involved in our operative reduction would require actually a personal demonstration, but a few points in philosophy: let me emphasise first the Harrington rods are shaped first to the shape of the normal spine, the fracture is operatively reduced by traction and by insertion of the Harrington rod into the upper hook then it is grasped with a clasp which I have designed and is not on the market. The lower end of the rod is forced downward, the gibbus is totally eliminated, then the rod is pulled into the lower hook while keeping the upper hook where it belongs. So we never accommodate the device to the fracture as it exists, we always anatomically reduce the fracture by first contouring the rod to the shape of the normal spine. I think that's important. Now there are distinct deficiencies in the Harrington system and I don't want everybody to think I am wedded to it although Paul Harrington is a K.U. graduate. I am over here in Europe for a sabbatical year and I am designing a new type of instrumentation similar to Harrington but eliminating many of the deficiencies in the Harrington. So we think there is progress to be made in this area.

DR P. MeYer. Responding to Dr Weiss's statements. First of all I think it is very important for this group to appreciate that as close friends as Dr Weiss and I are, we do 
not agree in everything. That makes it a very honest discussion. Secondly, it is important to report to you something which is not demonstrated to you on the slides but it is in my paper. The 349 cases reported here with fracture dislocations, four patients deteriorated neurologically who were treated conservatively. Now that is not to say that the 22 who had closed manipulation and reduction were very good because we were just lucky, but it does point out that even with conservative care-and I am waiting for Sir Ludwig to get up-I would anticipate that some patients will deteriorate even with the best conservative postural reduction management from pure loss of vascular supply to segments of the cord.

Sir GEorge BedBrook (Australia). Living as I do in an area of a muscle pathologist, I have naturally had to spend a bit more time looking at muscle pathology; and my reviews of the literature, which are now fairly extensive, show me that very few people have talked about the muscle problems of the spinal column in relationship to these injuries. We have looked at this just a little, yet we know so little about the posterior primary ramus. We know that it supplies muscle tissue at least two segments below the spinal column of injury, in fact we think it may supply tissue at least four segments below the spinal injury, and Bill Donovan has just recently finished an EMG study on some of the older cases and we have found neurological activity five segments below the spinal injury. Now this has not been recorded nor is it commented on by any of the workers who advocate internal fixation. We have looked at a few cases that have been internally fixed or internally splinted, they certainly have not got that neurological pattern. Whether this will be a temporary loss or whether it is a permanent loss I can't answer, I rather think some of it is permanent.

The second comment is that in looking very carefully through the literature on spinal fusion in relation to Harringtons, I was pleased to note that Dr Jacobs got his average spinal fusion down to $\mathrm{I} \cdot 45$ segments. The conservative group get them down to about $\mathrm{I}$ intervertebral segment; and I am concerned about the mobility of the spinal column and nobody talks about it. We've tried to find a method of evaluating spinal movements. The patients that I have seen both in Western Australia and in Sydney do tell me how much better they feel when the rods are out, and I think we should listen to patients just occasionally. Now come the two questions, and I must say how interested I was to listen to Paul Meyer's paper. I don't want to comment on it although I certainly wouldn't use that particular method, but I have a little experience in using Böhler's method and I must say that in particular cases we have been satisfied with it. Now I'd like to ask, particularly Dr Jacobs, he made absolutely no reference at all to the problem of urinary tract infection and again the literature is replete with gentlemen who tell me how they manage the spinal column, but nobody talks about the concomitant management of the urinary tract and I know very well that most of these people have got at least 50 per cent urinary infection. Now no self-respecting orthopaedic surgeon would operate on a patient with infection, but these patients are operated on. Secondly, nobody talks about the spinal mobility and I want to ask Dr Jacobs the question: would he tell us about the spinal mobility of his patients not at the time of operation but in 6 months time; has he photographed them; has he looked at them being mobile in a wheelchair and so on? I've talked for too long, but I have looked at this in very great depth and I think we should look at the whole spectrum. Too many people are looking at the middle range of the spectrum and not looking from where to go, as we say in Australia, and I'd like Dr Jacobs to answer those two questions. His urinary infection and his complication rate were really very low. I regret to tell him that most of the literature series have a complication rate of about i 5 per cent and this was shown by Munro a long time ago.

DR JACOBS (U.S.A.). First the percentages on complications: is per cent in the recumbent group and 7 per cent in the elevatory treated group. As to urinary tract infection, first I'll say that we are all aware that this whole problem of spinal injury is tremendous. Our paper concentrates on the possibility of mobilising patients early by rigid internal fixation. It may well be, although we have never thought of it, that urinary tract infections could possibly be decreased by this procedure, by getting patients upright and promoting urinary flow, I don't know; but that was not the thesis of the paper, 
but I feel for a comprehensive paper it should be included. As to your last question of spinal mobility: we have not measured every case that relates to spinal mobility. I question exactly how we can measure it. This particular patient will show you there is good muscle development certainly down to the level of the lesion. We feel it is important to preserve perispinous muscle activity. Our technique allows very minimal stripping of the muscles off the spinous processus just to the lamina. We feel that by doing an unnecessary spinal fusion you will probably get a much greater injury of muscle, therefore we avoid spinal fusion and only do it when we feel that it's necessary. Our technique is to put in as I said long Harrington rods that are very close to the midline and to do a spinal fusion only when the posterior ligaments are injured. The rods all come out by $\mathrm{I}$ year. At 3 months after the rods come out and after the patient has been on a rehabilitation programme to strengthen the paraspinous muscles both before and after rod removal. We then do flexion extension films of his spine to assure ourselves that he is stable at the site of injury and that he has normal mobility above and below the spine. Thus far we have normal mobility above and below. Also, another question that may be brought out. What in the world happens to the spine when you immobilise it with a Harrington rod without a fusion and then you take the rod out at the end of the year, have you possibly injured the facets or injured the spine in some way? Thus far all we can say is that the $\mathrm{X}$-rays show normal motion in range of flexion extension number one and number two, we have had no complaints from patients whatsoever as to pain in the spine.

DR Young. Dr Nuseibeh has been most patient, so we will hear from him and then you and then Sir Ludwig.

DR NUSEIBEH. You were asked about operating when there is urinary tract infection.

DR Young. I know, but that's all right.

DR JACOBS. I said I didn't have the data about urinary tract infection.

DR NUSEIBEH. You were asked about operating when there is urinary tract infection.

DR JACOBS. Oh no, the answer to that is no. In fact our thesis is that we operate early, before urinary infection sets in. We want to operate within 24 hours, that is our goal.

DR I. NuseibeH (G.B.). In support of Sir George I would like to comment on Dr Jacobs' paper. It is not lateral bending or even hyperextension that is important, it is the flexion which is important. I have to explain it and put it in simple words. I have patients who came to me and said 'We can't put on our socks, we can't put our shoes on, we can't put our trousers on; so what does it mean? It means although we have acquired nice alignment in X-ray we have interfered with his rehabilitation and early rehabilitation. Now a second point which I would like to ask Dr Meyer. It always worries me, this closed manipulation, since the big vessels are lying on the vertebral column.

DR MEYER. The importance of giving a medical paper is to describe not only the successes but the complications and to maintain integrity by honesty. In all honesty I am very concerned each time I do a closed manipulation and I think of everything that you've thought of and probably some other things that I haven't even thought of that you've thought of. I have attempted to do the procedure in patients whom I felt would not be neurologically injured or made worse with the manipulation. The vascular injury should occur with the initial injury to the spine. The injury to the spine initially is thousands of pounds greater than the manipulative procedure and so the injury to the vascular system is something that worries me more with surgery than it does with the manipulation.

Sir Ludwig Guttmann (G.B.). I am quite relieved to hear our colleagues who advocate surgical procedures, how they differ in their techniques. This shows that no surgical method is really accepted as a standard method. If you operate you have to see the whole patient as Bedbrook rightly demands, and the infection which follows whether you operate immediately or not. You follow up your patient, don't you, and if you will tell me by this kind of technique you use that the patient has sterile urine I don't believe you, unless you have done non-touch intermittent catheterisation as it should be done. With regard to these various X-rays you have shown, Dr Jacobs. They are not satisfactory because you have shown the X-rays just immediately after the operation and not follow-up 
$\mathrm{X}$-rays 4 months, 6 months later; and then we will see, Dr Jacobs, whether the Harrington rod is really the best method or any other surgical method as Marian Weiss has said. With regard to Paul Meyer, I am very glad he has now followed up the conservative approach. What you are doing really is not manipulation it is a forced reduction which has been introduced first by Hippocrates and later by the French surgeon, Calot. He used exactly the same method, if you look at his initial photograph as reproduced in my book and you have shown in your illustration, you will see that you are doing the same, which is in my opinion a forced reduction. I still advocate reduction by postural reduction and this is best done in the supine position. With regard to Dr Meyer's point that by conservative treatment of any kind, including my postural reduction, in a few instances deterioration may occur. This is of course well known and I have described these few cases in my previous publications (1963, 1976). These few cases of deterioration compare most favourably with the deleterious consequences following immediate surgary, and I refer to the most important papers of Morgan and recently of Bernard Sussman.

DR MEYER. I do not advocate this procedure. I'd like to end my statement by saying I do not advocate it. It is strictly from an academic viewpoint.

SIR LUDWIG. Not forced reduction, or what you call manipulation, but a gentle gradual reduction and you will have the same result if not better. And finally to Dr Jacobs, you talked about recumbency, what do you mean by recumbency? That is a method which is completely abandoned. I would be glad if you would explain what you mean with recumbency. Do you let the patient lie only in the supine position without regular turning? That would lead to, in addition to pressure sores, the development of ascending urinary infection, stones, etc.

DR Young. One brief definition of recumbency.

DR JACOBS. Recumbency is as described on the slide, the patient is placed in an ordinary hospital bed and nursed in the usual manner with turning and positioning pillows.

DR Young. Thank you, panel. And we will now proceed with the last two papers of the day. As I told you, this is a non-controversial subject. 